\title{
Aesthetic Rehabilitation in a Young Boy with Non-Syndromic Oligodontia: 2 Year Follow Up. A Case Report
}

\section{Valentine Rosadi Sinaga*}

Department of Prosthodontics, Faculty of Dentistry, Universitas Padjajaran, Bandung, Indonesia

*Corresponding Author: Valentine Rosadi Sinaga, Department of Prosthodontics, Faculty of Dentistry, Universitas Padjajaran, Bandung, Indonesia.
Received: December 27, 2021

Published: January 31, 2022

(C) All rights are reserved by Valentine Rosadi Sinaga.

\begin{abstract}
A 10-year-old boy with oligodontia came to the dental practice with the main complaint about facial appearance. He feels insecure about making friends in school, since his friends always ask about how did his teeth become so weird. The boy was diagnosed with nonsyndromic oligodontia, had several deciduous and permanent teeth. To help with the alignment of the tooth, the boy was referred to an orthodontist and used braces for a year. After that, a denture was made to raise the vertical height of the jaw and finalized with the reshaping of the incisive teeth with direct composite. The patient was happy with the denture, and we continue to check every six months to adjust the denture.
\end{abstract}

Keywords: Oligodontia; Hypodontia; Prosthetic; Denture

\section{Introduction}

Dental disharmony is a problem caused by defective or excessive teeth number resulted in functional and aesthetic alterations [1]. Tooth agenesis is described as the developmental absence of at least one deciduous or permanent teeth, excluding the third molars. The term Hypodontia is a condition as a consequence of tooth agenesis. It is the most common deformity in humans [2,3] and can happen as nonsyndromic isolated portion or as part of a known characteristic syndrome [3]. Oligodontia means that six or more teeth are congenitally absent, on the other hand anodontia refers to the lack of teeth at birth $[1,4]$.

Genetic, traumatic, nutritional, hereditary, infectious, and viral are some of the etiology factors of congenital tooth absence, with the latter becoming the main etiologic component explored in this study. Several prior studies examined the link between tooth agenesis and genetic abnormalities in members of the same family, focusing on MSX1, AXIN2, EDA, and PAX9 mutations [4].
Between 1.6 percent and 9.6 percent of people have agenesis of one or more teeth in the same dentition (permanent or deciduous). In European, the prevalence is between 0.4 and 0.9 percent, while in the global population, it is between 2.3 and $10 \%$. On the other hand, agenesis in the deciduous dentition was discovered to be less common (0.08-1.55\%) [3].

The prevalence of agenesis is described in the literature by nation and gender (male/female) and it was found to be higher in Europe (4.6\% males, 6.3\% females) and Australia (5.5\% males, 7.6\% females) than in the North American Caucasian population $(3.2 \%$ males, $4.6 \%$ females). Furthermore, females also have a prevalence of 1.37 times that of males [1].

Based on the aforementioned elaboration, this case report seeks to present a distinguishing feature oligodontia, which can occur in both primary and permanent dentition and is unrelated to any syndrome. Specifically, this study is discussing development of 10 years-old boy teeth, its treatment during the period of the study, and the follow up assessment. 


\section{Case Report}

A ten-year-old Indonesian boy consulted to private dental clinic complaining about his insecurities to socialize. He has no difficulties in mastication but feels less of self-confidence to make friends in class. His parents explained a positive history that there were no associated prenatal, birth or medical condition, additionally pediatrician examination and growth evaluation were conducted. It was found that the boy was normal and has no aberrant underlying systemic diseases. However, the extra-oral examination showed that the lower part of the boy face's vertical dimension was decreased, resulting in elderly-looking appearance. Moreover, the intraoral examination revealed mixed dentition with full erupted in the first permanent molar in all regions. The panoramic x-rays show the presence of first incisive in the left region of maxilla and one premolar tooth bud in each side. Both of the lower permanent canines are presence, along with second incisive in the right mandibular. Also, the salivary secretion and the teeth structure were normal. Following a comprehensive clinical and radiological assessment, removing denture was suggested and to be followed by teeth rehabilitation using Osseo-integrated implants was discussed with the parents.

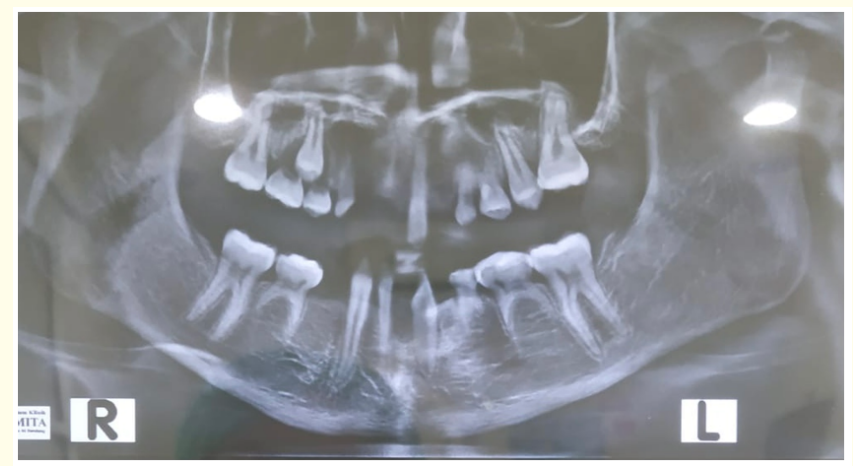

Figure 1: The panoramic x-ray taken as initial condition at 10 years of age.

As a preparation to fulfil the aesthetic goal with removable denture, incisive deciduous teeth in the maxilla and mandible were extracted. All of the teeth were broken and unfavorable. The lower left permanent canine was impacted and only shown a little in the gum. Orthodontic braces were used for a year to move the teeth bodily. A panoramic x-ray examination was done a year later and revealed the lower left canine and upper left premolar fully erupted into the arch therefore the deciduous molar teeth were extracted. The right deciduous upper molars were mobile therefore it was also extracted. The remaining oral cavity features were normal.

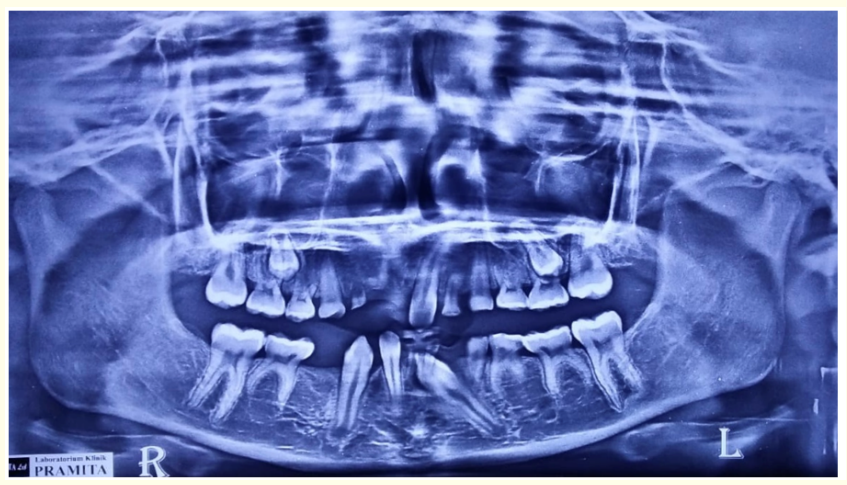

Figure 2: The panoramic x-ray one and half year later after orthodontic braces was used to align the teeth. Several deciduous teeth were extracted due to unfavorable condition.

Preliminary impression was made using irreversible hydrocolloid and, on the models, custom impression trays were created using auto-polymerizing acrylic resin. After that, the green-stick compound was used to border-molding the custom trays. The secondary impression was made using light-body polyvinylsiloxane paste. To create final casts, Type-IV dental stone was poured. Occlusal rim was used to record the bases and jaw relation. Next, the artificial acrylic teeth were positioned on the articulated wax rims and on a semi-adjustable articulator, a bilateral balanced occlusion was produced. Finally, maxillary and mandibular trial denture bases in the patient's mouth and vertical dimension at occlusion were checked.

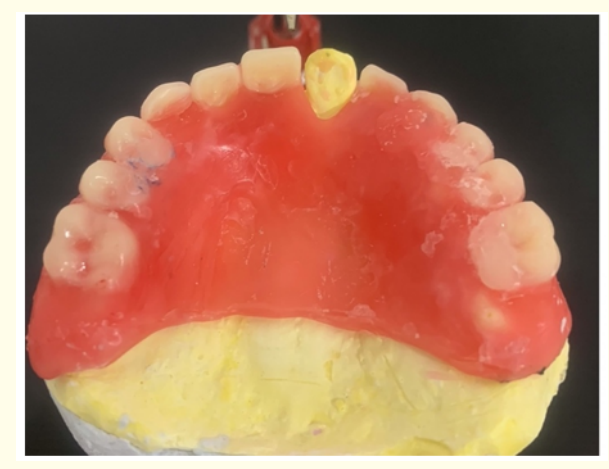




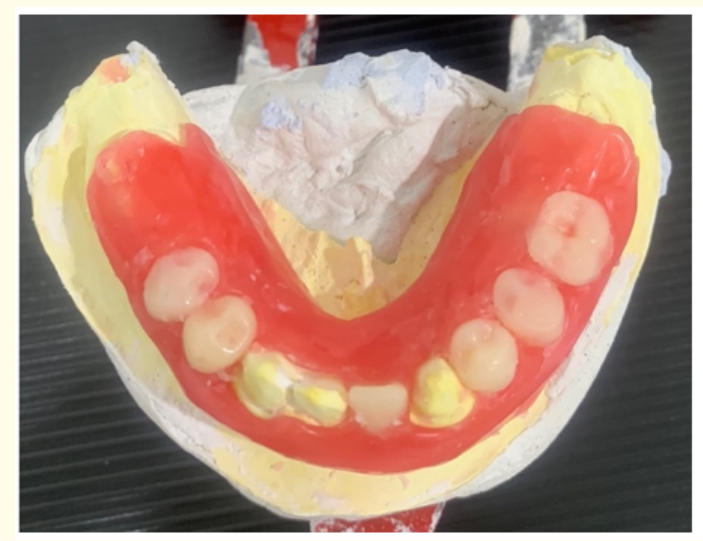

Figure 3: The trial denture bases after vertical dimension at occlusion was verified. This basis was tried in the patient's mouth.

The waxed dentures were manufactured in a heat-cured acrylic resin base lined with soft acrylic resin material in the anatomic surface after the patient and parents approved the trial denture bases. The completed dentures were put in the patient's mouth with the reshaping of the upper left first incisive to imitate the artificial teeth using composite resin as the prostheses were finished and cleaned. The patient and the parents got details instruction on oral tissues and prosthesis maintenance.
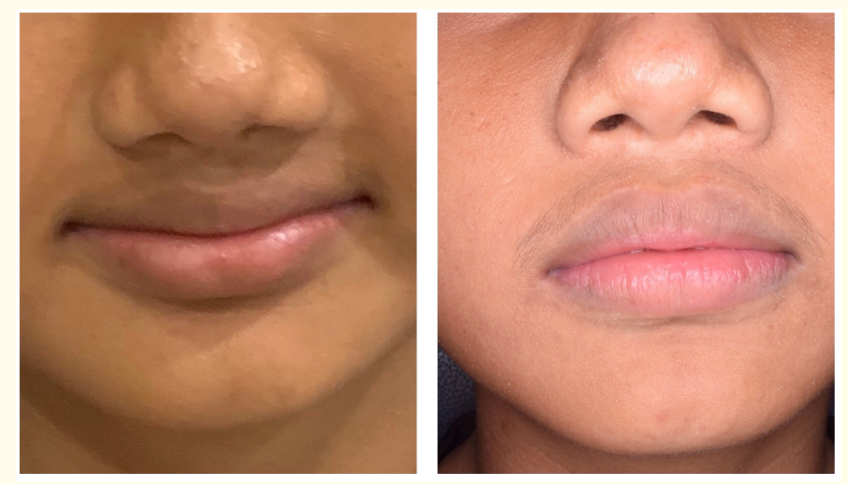

Figure 4: The photo of the patient's lips. (A) Initial condition before using dentures. (B) The condition after patient uses the dentures. The lips were fuller and the vertical dimension was established at the right height.

The patient was told to schedule the adjustments in a week, followed by three months regular checkup to modify and/or replace

the prosthesis. The pictures of dentures in place illustrate the improvement on appearance harmony, speech and masticatory The eruption of upper right premolar, with a following up on sanitary measures and topical fluoride applications.

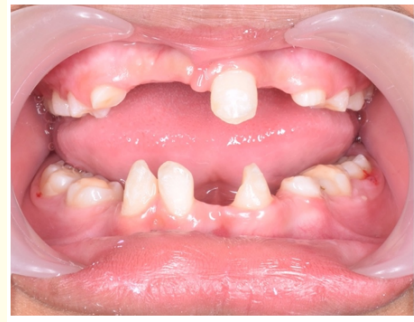

Figure 5: The intraoral photos. (A) Initial condition (B) With dentures and reshaping of the first incisive upper teeth, optimum aesthetic was achieved.

\section{Discussion}

Hypodontia has an functional, financial burden, psychosocial, and aesthetic for the affected. Hypodontia can be a long-term condition that necessitates meticulous treatment planning to achieve the greatest results. In this case, a long-term maintenance and family counseling are required for the treatment method. On the other hand, from the initial visit to the completion, hypodontia treatment can take many years. Furthermore, jaws and alveolar bone hypodevelopment implicates facial structures modification with aesthetic and functional consequences [5].

In this case we found that the young boy looks older as he has less vertical height, also the first incisive permanent teeth smaller than the common size (microdontia). This feature is common in oligodontia children as it is widely reported in case reports $[3,6]$.

Complaint's assessment becomes important for the patient and the parents since the treatment planification and support from patient and family are crucial for the rehabilitation. In order to make it more optimal, agenesis should be identified as early as possible throughout childhood, and treatment should begin with the placement of a paediatric prosthesis as soon as the kid gives his or her approval [5,7].

In these reports, the young boy didn't have a prosthesis before since the unawareness of facial and mastication disturbances by the parents and the patient. Presumably, the lack of some perma- 
nent teeth has negative impact on the OHrQoL (Oral Health Quality in Life) of those aged between 11-15 years. This is in agreement with Filius., et al. [8] stating that eating and speaking (in public) are not affected by oligodontia, considering that they have not missed their deciduous teeth. Patients who had lost their deciduous teeth and had no successors experienced chewing difficulty. Authors also stated the awareness about appearance during puberty raise in young oligodontia patient (11-12 years of age) and the OHrQol assessment was substantially inferior in patients above 12 years old compared to the younger patients.

The single examples of oligodontia were inherited as an autosummoned dominant trait with incomplete penetrance and variable expression, with no genetic alterations. In families with nonsyndromic familial oligodontia, mutations in MSX1 and PAX9 have been discovered $[3,9]$.

A series of treatment method had to deal with the missing teeth of hypodontia patients. Due to its complexity, an interdisciplinary method was required in which the financial cost becomes consideration for both patient and their family. Thus, adept dental specialists should be involved during the treatment. There are four phases of the global treatment including removable prosthesis during the growth, orthodontic treatment, pre-prosthetic surgery, and implant-borne rehabilitation [5].

Early identification is critical for preventing aesthetic and functional issues in dentition care, especially when early prosthetic reconstruction is required. There is a significant delay in dental age in patients with Oligodontia and it will affect the treatment planning [10]. It should provide the patient with temporary solution to address the aesthetic and functional requirement and to keep tissue from being damaged unnecessarily. A prosthodontics rehabilitation is fundamental to provide a functional and aesthetic solution to help a child live as normal as possible without the damaging his/ her self-esteem or physiological development [9].

The impact of physiological and mastication disturbances in oligodontia children may appear to be higher in nations where oligodontia dental treatment is not covered [7]. In this case, oligodontia therapy is not covered by insurance in Indonesia. So, in the future, patient with oligodontia may get some benefit from using dental implants and therefore need financial planning.

\section{Conclusion}

Children with oligodontia need full assessment and awareness from parents and physicians about different aspect of their life that would be affected with this condition such as function and psychosocial well-being due to the cosmetic, functional, and economical load. Therefore, the right timing of treatment by comprehensive multi-disciplinary will keep their quality of life.

\section{Funding}

This work was not supported by grant from anyone.

\section{Conflict of Interest}

The author has no conflict of interest to disclose.

\section{Bibliography}

1. Galluccio G., et al. "Genetic basis of non-syndromic anomalies of human tooth number". Archives of Oral Biology 57.7 (2012): 918-930.

2. Coelho Neto OL., et al. "Clinical and Genetic Analysis of a Nonsyndromic Oligodontia in a Child". Case Reports in Dentistry 2014 (2014): 1-5.

3. Al-Ani AH., et al. "Hypodontia: An Update on Its Etiology, Classification, and Clinical Management". BioMed Research International (2017).

4. Coelho Neto OL., et al. "Clinical and Genetic Analysis of a Nonsyndromic Oligodontia in a Child. Adeyemo WL, editor". Case Reports in Dentistry 2014 (2014): 137621.

5. Lauwers L., et al. "11 - Implants in congenital missing teeth. In: Ferri J, Hunziker EBBT-P and MS, editors". Woodhead Publishing Series in Biomaterials (2011): 185-198.

6. Ahmed R., et al. "Prosthetic rehabilitation of oligodontia in a child: a case report". Update Dental College Journal (2014).

7. Hobkirk JA., et al. "Hypodontia: A Team Approach to Management” (2013): 1-199.

8. Filius MAP., et al. "Oral health-related quality of life in dutch children diagnosed with oligodontia. A cross-sectional study". International Journal of Environmental Research and Public Health 16.13 (2019).

9. Moses J., et al. "Non-syndromic oligodontia of primary and permanent dentition: 5 year follow up-a rare case report". Journal of Clinical and Diagnostic Research 7.4 (2013): 776-779. 
10. Ruiz-Mealin EV., et al. "Radiographic study of delayed tooth development in patients with dental agenesis". American Journal of Orthodontics and Dentofacial Orthopedics 141.3 (2012): 307-314.

\section{Assets from publication with us}

- Prompt Acknowledgement after receiving the article

- Thorough Double blinded peer review

- Rapid Publication

- Issue of Publication Certificate

- High visibility of your Published work

Website: www.actascientific.com/

Submit Article: www.actascientific.com/submission.php

Email us: editor@actascientific.com

Contact us: +919182824667 